\title{
Unperturbed Dimensions of Vinyl Polymer Chains*
}

\author{
Akihiro ABE \\ Central Research Laboratory, Showa Denko Co., 2-24-60 Tamagawa, Ohta-ku, Tokyo, Japan.
}

(Received December 11, 1969)

\begin{abstract}
The unperturbed dimensions of poly- $\alpha$-olefins are treated in consideration of the side chain configuration. Within the reasonable range of conformational energies, the root mean square end-to-end distance $\left\langle r^{2}\right\rangle_{0} / n l^{2}$ and its thermal coefficient $d \ln \left\langle r^{2}\right\rangle_{0} / d T$ are evaluated and compared with those observed. The presence of several percent of heterotactic units in an allegedly isotactic poly-1-butene or poly-1-pentene is the primary requirement in finding the agreement between theory and experiments. The conclusion drawn in a previous paper is thus reconfirmed. When the pendant group is methyl, on the other hand, the experimental value of $\left\langle r^{2}\right\rangle_{0} / n l^{2}$ is found to be consistent with those calculated over a wider range of isotacticity $(0.92-0.98)$, provided that the thermal coefficient is negative and large. The treatment is further extended to the polymer systems with bulky substituents. It is pointed out, finally, that the magnitude of $\left\langle r^{2}\right\rangle_{0} / n l^{2}$ cannot provide a good criterion to define the stiffness of the vinyl chain system except when the polymer possesses a stereoregular configuration.

KEY WORDS Unperturbed Dimension / Configuration / Confor-
mation / Chain Stiffness / Poly- $\alpha$-olefin / Pendant Group /
Isotacticity /
\end{abstract}

Stereoregularity of highly crystalline polypropylene prepared by the Ziegler-Natta catalyst has been a topic of recent argument ${ }^{1-4}$. In our previous studies on the unperturbed dimension ${ }^{1}$, presence of several percent of heterotactic placements in an allegedly isotactic poly- $\alpha$-olefin chain was postulated. On such assumption, the agreement was found between theoretical calculations and experimental observations on isotactic poly1-butene and poly-1-pentene, for which values of $\left\langle r^{2}\right\rangle_{0} / n l^{2} \cong 10$ and $d \ln \left\langle r^{2}\right\rangle_{0} / d T \cong 0$ seem to have been established ${ }^{5,6}$. Lately Zambelli, Segre, Farina and $\mathrm{Natta}^{2}$ concluded from the NMR investigation on variously deuterated polypropylene samples that the isotacticity for the heptane insoluble fraction is more than $98 \%$. Based on the studies on configurational statistics of vinyl polymer systems, however, Flory and Fujiwara ${ }^{4}$ pointed out the inapplicability of the NMR method to the quantitative detection of a small fraction of heterotactic units when buried in highly isotactic polymer sequences.

From the crystallographic point of view, Natta and Corradini ${ }^{7}$ raised an objection to the chain model employed in our calculations ${ }^{1}$, which assumes the Bernoullian distribution of isotactic

\footnotetext{
* Presented at the 18th annual meeting of the Society of Polymer Science, Japan, Kyoto 1969.
}

and syndiotactic dyads along a polymer chain. As they pointed out, the heterotactic placements which can be incorporated in a highly crystalline isotactic polymer may be limited in their stereochemical forms as well as in numbers. In fact, another chain model comprising stereoirregularity of the type $d l d$ or $l d l$ dispersed randomly among the isotactic $d$ or $l$ chain, respectively, has been examined in the study of the optical rotatory properties of poly- $\alpha$-olefins $s^{8}$ or in the theoretical treatment of the unperturbed dimension of polypropylene?

On the other hand, from the viscometric determination in a series of theta solvents, Nakajima and Saijo ${ }^{10}$ suggested a large negative thermal coefficient of the unperturbed dimension for highly isotactic polypropylene. Such advancement in experiments provides a further basis to pursue a more probable concensus among these apparently divergent views by reevaluating the unperturbed mean square end-to-end distance with steric interaction parameters carefully chosen.

Vinyl polymer chains such as<smiles>[R]C(CC(C)C)C(C)(C)[N]</smiles>

are treated in the framework of the trans-gauche 
three rotational isomeric state scheme ${ }^{1,11}$ with emphasis on the steric effect imposed by the spatial arrangements of the side chains.

\section{CONFORMATIONAL ANALYSIS}

A portion of an isotactic $d$ chain is depicted in Figure 1. The statistical weight matrices $U^{\prime}$ defined for a bond like $i$ have been given in a generalized form $^{8}$ by

$$
\begin{aligned}
U_{d}{ }^{\prime} & =\left[\begin{array}{ll}
\beta(t, t) & \beta\left(t, g^{+}\right) \\
\beta\left(g^{-}, t\right) & \omega^{\prime \prime} \beta\left(g^{-}, g^{+}\right)
\end{array}\right] \\
U_{l}{ }^{\prime} & =\left[\begin{array}{ll}
\beta(t, t) & \beta\left(t, g^{-}\right) \\
\beta\left(g^{+}, t\right) & \omega^{\prime \prime} \beta\left(g^{+}, g^{-}\right)
\end{array}\right]
\end{aligned}
$$

where the subscripts $d$ and $l$ indicate the configuration of the pseudo-asymmetric tertiary carbon in the main chain. The conformational statistical weight factors $\beta(\zeta, \eta)$ in Eq. 1 represent the contribution from the side chain when the adjoining skeletal bonds $i-1$ and $i$ are in the rotational states of $\zeta$ and $\eta$, respectively. The rotational isomeric states of $t, g^{+}$and $g^{-}$are defined to occur at the rotational angles about $0,(2 / 3) \pi$ and $(4 / 3) \pi$, respectively, by the clockwise rotation around the rear carbon as expressed in the Newman projection. Contribution from the high energy conformation designated previously with $\omega^{\prime}$ is practically negligible ${ }^{1,8}$. Then the order of the statistical weight matrices can be reduced to a $2 \times 2$ matrix scheme as given in Eq. 1 for the trans-gauche three rotational state model.

For polypropylene where $\mathrm{R}=\mathrm{CH}_{3}, \beta(\zeta, \eta) \cong$ 1 for any set of $\zeta$ and $\eta$. Thus we have

$$
U_{d}{ }^{\prime}=U_{l}^{\prime}=\left[\begin{array}{cc}
1 & 1 \\
1 & \omega^{\prime \prime}
\end{array}\right]
$$

From the symmetry consideration, the equality $U_{d}{ }^{\prime}=U_{l}{ }^{\prime}$ should be valid for any vinyl polymer with symmetric side chains*. The corresponding matrices for some higher $\alpha$-olefin polymers are given in Eqs. 3 and 4.

$$
\begin{aligned}
& \mathrm{R}=-\mathrm{CH}_{2} \mathrm{CH}_{3} \\
& U_{d}{ }^{\prime}=\left[\begin{array}{lc}
\tau+2 \omega^{\prime \prime} & 1+\tau+\omega^{\prime \prime} \\
1+\tau+\omega^{\prime \prime} & (2+\tau) \omega^{\prime \prime}
\end{array}\right]
\end{aligned}
$$

* When the side chain contains an asymmetric center, $U_{l}^{\prime}$ should be given as the transpose of $U_{d^{\prime}}$. See ref. 8 .

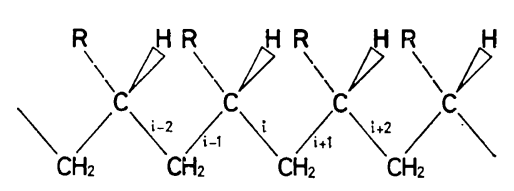

Figure 1. Schematic representation of a vinyl chain in the planar conformation. Defined as a $d$ chain when viewed from left to right.

$$
\begin{aligned}
& \mathrm{R}=-\mathrm{CH}_{2} \mathrm{CH}_{2} \mathrm{CH}_{3} \\
& U_{d}{ }^{\prime}=\left[\begin{array}{cc}
\tau+2(1+\sigma) \omega^{\prime \prime} & 1+\sigma+\tau \\
1+\sigma+\tau & +(1+2 \sigma) \omega^{\prime \prime} \\
+(1+2 \sigma) \omega^{\prime \prime} & (2+2 \sigma+\tau) \omega^{\prime \prime}
\end{array}\right]
\end{aligned}
$$

Here notations $\sigma, \tau$ and $\omega^{\prime \prime}$ are employed in keeping with the previous usage ${ }^{1,8}$ to represent the conformational statistical weight factors characteristic to the three-bond and four-bond interactions. Since we neglect the long range interactions beyond those given above, the statistical weight matrices $U^{\prime \prime}$ defined for a bond like $i+1$ shown in Figure 1 are unaffected by the side chain structure, and are, therefore, commonly applicable to any vinyl polymer system; ${ }^{1}$

$$
\begin{aligned}
& U_{d d}{ }^{\prime \prime}=U_{l l}{ }^{\prime \prime}=\left[\begin{array}{cc}
\omega^{\prime \prime} & 1 \\
1 & \omega^{\prime \prime}
\end{array}\right] \\
& U_{d l}{ }^{\prime \prime}=U_{l d}{ }^{\prime \prime}=\left[\begin{array}{cc}
1 & \omega^{\prime \prime} \\
\omega^{\prime \prime} & 1
\end{array}\right]
\end{aligned}
$$

The relative stability among various rotational isomeric states of hydrocarbons are generally studied by spectroscopic or by thermodynamic methods $^{12}$. Theoretical estimation of conformational energies based on the calculation in consideration of the torsional potentials intrinsic to $\mathrm{C}-\mathrm{C}$ bonds and the short-range interactions between nonbonded groups also provides important information. ${ }^{13,14}$ For $n$-alkanes, in the case where all these methods are in agreement, ${ }^{14}$ we have

$$
E_{\sigma}=-R T \ln \sigma \cong 500 \mathrm{cal} \cdot \mathrm{mol}^{-1}
$$

Experimental results for the conformational energy difference between two conformers of 2-methylbutane $\mathrm{e}^{15,16}$ are quite divergent, and the real value is still in question. Calculations ${ }^{1,14}$ suggest this energy difference to be of the order of $1 \mathrm{kcal} \cdot \mathrm{mol}^{-1}$. For the present purpose, the 
enthalpy difference between the axial and the equatorial methylcyclohexane is also suggestive because steric interactions responsible for this energy difference are similar to those involved in 2-methylbutane conformers. The experimental value $^{17}$ was reported to be 1.6 to $1.8 \mathrm{kcal} \cdot \mathrm{mol}^{-1}$ in the liquid state, and $1.9 \mathrm{kcal} \cdot \mathrm{mol}^{-1}$ in the gas phase. Altogether, we estimate

$$
E_{\tau}=-R T \ln \tau=1-2 \mathrm{kcal} \cdot \mathrm{mol}^{-1}
$$

No reliable experimental data are available for the highly repulsive four-bond interactions. The one realizable in the $\left(g^{ \pm}, g^{\mp}\right)$ state of $n$-pentane was estimated from the conformational calculations to be higher by $c a .3 \mathrm{kcal} \cdot \mathrm{mol}^{-1}$ than the $(t, t)$ conformation $^{14}$. This estimation was also supported from the analysis of the observed data on the unperturbed end-to-end distance of polyethylene. In a vinyl polymer system, the fourbond interactions designated with the statistical weight factor $\omega^{\prime \prime}$ are encountered, e.g., for a $d$ chain, when bonds like $i-1$ and $i$ are in the rotational state of $g^{-}$and $g^{+}$, respectively, and when bonds like $i$ and $i+1$ are both in the trans state or in the $g^{+}$and $g^{-}$states, respectively. These are illustrated in Figure 2. The conformations given in Figures 2(a) and (c) may be identified with the $\left(g^{ \pm}, g^{\mp}\right)$ conformation of polyethylene when $\mathrm{R}=\mathrm{H}$. With $\mathrm{R}=\mathrm{CH}_{3}$, however, the systems become more crowded and, consequently, the effect of mitigation of the highly repulsive interactions through the adjustment of internal rotation angles should be more or less suppressed. In Figure 2(a), three-bond interactions between $\mathrm{R}$ groups sticking out towards the rear and the $\mathrm{CH}$ group adjoining bonds $i-1$ and $i$, as distinguished by the underline, restrict the bond rotations about bonds $i-1$ and $i$, and to some extent, those about bonds $i-2$ and $i+1$, respectively. Similar interactions between $\mathrm{R}$ and $\mathrm{CH}$ groups in Figure $2 \mathrm{c}$ increase the steric hindrance to the rotations about bonds $i-1$ and $i+2$, respectively. No such three-bond interactions are present in the conformation shown in Figure 2(b) when $\mathrm{X}=\mathrm{H}$ for polypropylene. Here only the backbone methylene groups extending out on both sides may complicate the rotations about bonds $i$ and $i+1$, respectively. With $\mathrm{R}=\mathrm{CH}_{2} \mathrm{CH}_{3}$ in Figures 2(a) and (c), and $\mathrm{X}=\mathrm{CH}_{3}$ in Figure 2(b), as inspection of the model reveals, the number of
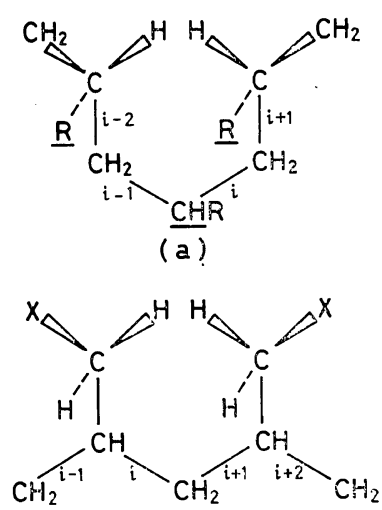

(b)

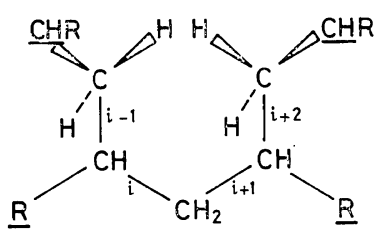

(c)

Figure 2. Four-bond interactions encountered in the vinyl polymer system. Conformations associated with (a) the $\left(g^{-} g^{+}\right)$for bonds $i-1$ and $i$, (b) the $(t t)$ for bonds $i$ and $i+1$, and (c) the $\left(g^{+} g^{-}\right)$for bonds $i$ and $i+1$ are depicted, respectively.

three-bond interactions unfavorable to the internal rotations about skeletal $\mathrm{C}-\mathrm{C}$ bonds increases still more, and therefore, the conformational energy expressed generally by $E_{\omega^{\prime \prime}}$ may be somewhat higher than that estimated for polyethylene. The introduction of longer alkyl side chains beyond ethyl (e.g., poly-1-pentene) does not add such short-range interferences as strengthen those four-bond conflicts discussed above. In polymer systems derived from 3- or 4-substituted $\alpha$-olefins, steric restraints which restrict the internal rotations may become a little more tight. In summary, we may assume the order for $E_{\omega^{\prime \prime}}$ as polyethylene $<$ polypropylene $<$ poly-1-butene and others, within the range

$$
E_{\omega^{\prime \prime}}=-R T \ln \omega^{\prime \prime}=2-5 \mathrm{kcal} \cdot \mathrm{mol}^{-1}
$$

These considerations permit an approximation for the statistical weight matrices $U_{d}{ }^{\prime}$ given in Eqs. 3 and 4 by a simplified form such as

$$
U_{d}^{\prime}=\left[\begin{array}{cc}
\tau^{*} & 1 \\
1 & \omega^{\prime \prime}
\end{array}\right]
$$


where the statistical weight factors are normalized to a weight of unity for the stable helical conformation*. Then $\tau^{*}=\tau-\tau^{2}+\ldots$ for the system with $\mathrm{R}=\mathrm{CH}_{2} \mathrm{CH}_{3}$, and $\tau^{*}=\tau-\sigma \tau-$ $\tau^{2}+\ldots$ for $\mathrm{R}=\mathrm{CH}_{2} \mathrm{CH}_{2} \mathrm{CH}_{3}$. A factor of two in the $(2,2)$ element of $U_{d}{ }^{\prime}$ in Eqs. 3 and 4 may be absorbed into $\omega^{\prime \prime}$. The statistical weight matrix given in Eq. 6 has a comprehensive form applicable to any vinyl polymer system depending upon the magnitude of $\tau^{*}$. When $\tau^{*}$ is replaced by unity, Eq. 6 becomes identical with Eq. 2 for $\mathrm{R}=\mathrm{CH}_{3}$. In a poly$\alpha$-olefin system where bond rotations are extremely hindered (e.g., poly(4-methyl-1-pentene) or poly(vinylcyclohexane)), $\tau^{*} \cong \omega^{\prime \prime}$ should be a valid approximation ${ }^{8,18}$. Williams and Flory $^{19}$ have studied stereochemical equilibrium among styrene oligomers, arriving at the conclusion that the trans-trans conformation is more stable by a factor of $\eta=0.5 \exp (350 / T), T$ being the absolute temperature, than the trans-gauche or gauche-trans helical arrangements for the two consecutive bonds on both sides of pseudo-asymmetric tertiary carbon. The system with $\mathrm{R}=$ phenyl can be represented in our scheme** by equating $\tau^{*}=\eta$ in Eq. 6 .

As discussed in a previous paper ${ }^{1}$, displacement of the rotation angles at the potential minima from the regular three-fold symmetry is another important factor when geometrical properties are considered. Conformational calculations based on 2,4-dimethylpentane as a model suggested that the displacement $\Delta \varphi$ could be about $20^{\circ}$ for the preferred helical conformation of vinyl polymer systems ${ }^{1}$. By analogy, we have also assumed previously that the rotational minimum should be displaced by $\Delta \varphi$ when consecutive bonds like $i-1$ and $i$ are both in the trans state. This latter assumption is however improbable for a vinyl polymer system with side chains like $\mathrm{R}=$ $\mathrm{CH}_{2} \mathrm{CH}_{3}$. In the conformation designated with the statistical weight $\tau$, displacement of the rotation angle by $\Delta \varphi$ about either of the adjoining skeletal $\mathrm{C}-\mathrm{C}$ bonds severely increases the three-bond steric repulsions. Such considerations lead to the assumption $\Delta \varphi \gg \Delta \varphi_{t}>0$ for bonds $i-1$ and $i$ when both are simultaneously in confor-

* The Eq. 6 thus derived will be identified with Eq. 7 of ref. 1 as expressed in the $3 \times 3$ matrix scheme. mations weighted by $\tau$. According to the model inspection, the rotational states about the neighboring bonds $i-2$ and $i+1$, respectively, may be perturbed similarly, but to a lesser extent. In more complicated polymer systems having side chains branched at the $\alpha$ or $\beta$ position with respect to the backbone chain, the internal adjustment of rotation angles is also important as may be judged from their solid state structure ${ }^{20}$. The magnitude of $\Delta \varphi$ for the preferred helical conformation probably is less than that of simple vinyl polymer systems.

Rotational minima for the four-bond interactions indicated by $\omega^{\prime \prime}$ in Figure 2 should be considerably displaced on account of the high steric conflicts involved. Because the statistical weight factor $\omega^{\prime \prime}$ is quite small, detailed exploration on this point is not important, however.

** Adaption of Eqs. 5 and 6 to the polystyrene system may require some elaboration. A pair of statistical weight matrices defined by Eqs. 5 and 6 of ref. 19 for a meso dyad can be expressed in the simplified $2 \times 2$ scheme as

$$
U_{m}^{\prime \prime} U^{\prime}=\left[\begin{array}{cc}
\eta \omega^{\prime \prime} & 1 \\
\eta & \omega
\end{array}\right]\left[\begin{array}{cc}
\eta \tau^{*} & 1 \\
\eta & \omega
\end{array}\right]
$$

Internal transformation leads to

$$
\begin{aligned}
& =\left[\begin{array}{cc}
\omega^{\prime \prime} & 1 \\
1 & \omega
\end{array}\right]\left[\begin{array}{cc}
\eta & 0 \\
0 & 1
\end{array}\right]\left[\begin{array}{cc}
\eta \tau^{*} & 1 \\
\eta & \omega
\end{array}\right] \\
& =\left[\begin{array}{cc}
\omega^{\prime \prime} & 1 \\
1 & \omega
\end{array}\right]\left[\begin{array}{cc}
\eta^{2} \tau^{*} & \eta \\
\eta & \omega
\end{array}\right]
\end{aligned}
$$

By normalizing to a weight of unity for the helical conformation (i.e., $t g^{ \pm}$or $g^{\mp} t$ ), we now have

$$
U_{m}^{\prime \prime}=\left[\begin{array}{cc}
\omega^{\prime \prime} & 1 \\
1 & \omega
\end{array}\right] \text { and } U^{\prime}=\left[\begin{array}{cc}
\eta \tau^{*} & 1 \\
1 & \omega / \eta
\end{array}\right]
$$

According to Williams and Flory ${ }^{19}, \tau^{*} \cong 1$. We assume further that various high energy four-bond interactions in the system can be represented by a single parameter $\omega^{\prime \prime}$. Those include steric repulsions between groups such as $\mathrm{CH} \ldots \mathrm{CH}, \mathrm{CH}_{2} \ldots \mathrm{CH}_{2}$ and $\mathrm{C}_{6} \mathrm{H}_{5} \ldots \mathrm{C}_{6} \mathrm{H}_{5} . \quad$ A factor $1 / \eta$ of the $\left(g^{ \pm}, g^{\mp}\right)$ element of $U^{\prime}$ may be absorbed into $\omega^{\prime \prime}$. Then

$$
U_{m}^{\prime \prime}=\left[\begin{array}{cc}
\omega^{\prime \prime} & 1 \\
1 & \omega^{\prime \prime}
\end{array}\right] \text { and } U^{\prime}=\left[\begin{array}{cc}
\eta & 1 \\
1 & \omega^{\prime \prime}
\end{array}\right]
$$

When $\eta$ is replaced by $\tau^{*}$, these may be identified with the expression given by Eqs. 5 and 6 of the present paper. A similar conclusion will be derived for a racemic dyad. It should be noted here that the physical meaning of $\tau^{*}$ and $\omega^{\prime \prime}$ is different from that of Williams and Flory ${ }^{19}$. 


\section{A. $\mathrm{ABE}$}

\section{EVALUATION OF THE UNPERTURBED DIMENSION AND ITS TEMPERATURE COEFFICIENT}

Computations were carried out in a similar manner to that described in ref. 1. Some minor modifications were adopted however when dimensions of higher olefin polymers were treated.

The transformation matrices $T(\varphi)$, which perform the orthogonal transformation from a Cartesian coordinate system defined for a given bond to that for the preceding bond, are customarily ${ }^{11}$ written as

$$
T=\left[\begin{array}{ccc}
\cos \theta & \sin \theta & 0 \\
\sin \theta \cos \varphi & -\cos \theta \cos \varphi & \sin \varphi \\
\sin \theta \sin \varphi & -\cos \theta \sin \varphi & -\cos \varphi
\end{array}\right]
$$

where $\theta$ is the supplement of the bond angle, and $\varphi$ is specified by the rotational state of the bond under consideration. For simplicity, we assume $\theta=68^{\circ}$ throughout the paper ${ }^{1}$. Depending upon the configuration of the participating tertiary carbon, a proper set of $\varphi$ 's should be chosen for a given bond;

$$
\begin{aligned}
\varphi_{t} & =\Delta \varphi_{t} \\
\varphi_{g^{+}} & =(2 / 3) \pi-\Delta \varphi_{g}
\end{aligned}
$$

or

$$
\begin{aligned}
\varphi_{t} & =-\Delta \varphi_{t} \\
\varphi_{g^{-}} & =-(2 / 3) \pi+\Delta \varphi_{g}
\end{aligned}
$$

The statistical weight matrix $U$ and the transformation matrices $T(\varphi)$ thus defined for a given bond are arranged in the $g$ matrix in a manner specified by Eq. 7 of ref. 14 . Sequential products of $g$ matrices properly chosen for the polymer chain configuration then yield $\left\langle r^{2}\right\rangle_{0} / n l^{2}$ according to the procedure prescribed by Eq. 12 of ref. 1 .

For the polymer system with $U_{d}{ }^{\prime}(t, t)=U_{l}{ }^{\prime}(t, t)$ $=\tau^{*}$ as in the case of polybutene-1, we define the $G$ matrix for a pair of bonds like $i-1$ and $i$ as

$$
G=g_{i-1}^{\prime \prime} g_{i}^{\prime}
$$

Depending upon the stereochemical arrangements of the neighboring units, we have four matrix products such as $G_{d d}, G_{d l}, G_{l d}$, and $G_{l l}$. By this device, it becomes possible to accommodate the condition $\Delta \varphi_{t}=0$ in the transformation matrices $T\left(\varphi_{t}\right)$ for bonds like $i-1$ and $i$ when they are affiliated with the $(t, t)$ conformation. Of course, the transformation matrices $T\left(\varphi_{t}\right)$ associated with the low energy helical conformations such as $\ldots t$ ) $\left(g^{ \pm} t\right)\left(g^{ \pm} \ldots\right.$ or $\left.\ldots g^{ \pm}\right)\left(t g^{ \pm}\right)(t \ldots$ remain unchanged, and are allowed to take non-zero $\Delta \varphi_{t}\left(=\Delta \varphi_{g}\right)$. Sequential products of $G$ matrices now give $\left\langle r^{2}\right\rangle_{0} / n l^{2}$ pertinent for the system with $\mathrm{R}=$ $\left(\mathrm{CH}_{2}\right)_{z} \mathrm{CH}_{3}(z \geqq 1)$.

For a finite chain, special care should be taken for the chain terminals. The matrix $U_{1}$ associated with the first bond is the identity of the $2 \times 2$ order. The matrices $U_{2}{ }^{\prime}$ and $U_{n}{ }^{\prime}$ should be expressed in the diagonal form, because of the absence of the preceding or the following bond rotational states, respectively. In our present computations, these are replaced by the identity matrix, for simplicity. If the polymer chain is sufficiently long, the error arising from such simplification for the end groups may be negligible.

Thermal coefficients of $\left\langle r^{2}\right\rangle_{0}$ can be obtained from the relation

$$
\begin{aligned}
\frac{d \ln \left\langle r^{2}\right\rangle_{0}}{d \ln T}= & -\ln \omega^{\prime \prime}\left(\partial \ln \left\langle r^{2}\right\rangle_{0} / \partial \ln \omega^{\prime \prime}\right) \\
& -\ln \tau^{*}\left(\partial \ln \left\langle r^{2}\right\rangle_{0} / \partial \ln \tau^{*}\right)
\end{aligned}
$$

by varying $\omega^{\prime \prime}$ and $\tau^{*}$ about their specified values to estimate the coefficients $\partial \ln \left\langle r^{2}\right\rangle_{0} / \partial \ln \omega^{\prime \prime}$ and $\partial \ln \left\langle r^{2}\right\rangle_{0} / \partial \ln \tau^{*}$, respectively. The second term on the right-hand side of Eq. 11 is of course unimportant in the case of polypropylene.

\section{NUMERICAL RESULTS}

For polymer systems with $\mathrm{R}=\mathrm{CH}_{3}$, an extensive study was carried out previously ${ }^{1}$. Variation of the characteristic ratio $\left\langle r^{2}\right\rangle_{0} / n l^{2}$ with $-\ln \omega^{\prime \prime}=E_{\omega^{\prime \prime}} / R T$ is reproduced for a perfectly isotactic chain* with $n=\infty$ in Figure 3, wherein also included are the results derived by taking $\tau^{*}<1$. For any given value of $\Delta \varphi$, the characteristic ratio $\left\langle r^{2}\right\rangle_{0} / n l^{2}$ increases very rapidly with $-\ln \omega^{\prime \prime}$, suggesting large negative values of the coefficient $\partial \ln \left\langle r^{2}\right\rangle_{0} / \partial \ln \omega^{\prime \prime}$. The slope of the curve is steeper for a smaller value of $\tau^{*}$ (i.e., a larger value of $\left.E_{\tau} * / R T\right)$. The similar effect of

* Since this polymer chain has a regularly repeated chain configuration, computations were carried out by the eigen-value method. See ref. 11 . 


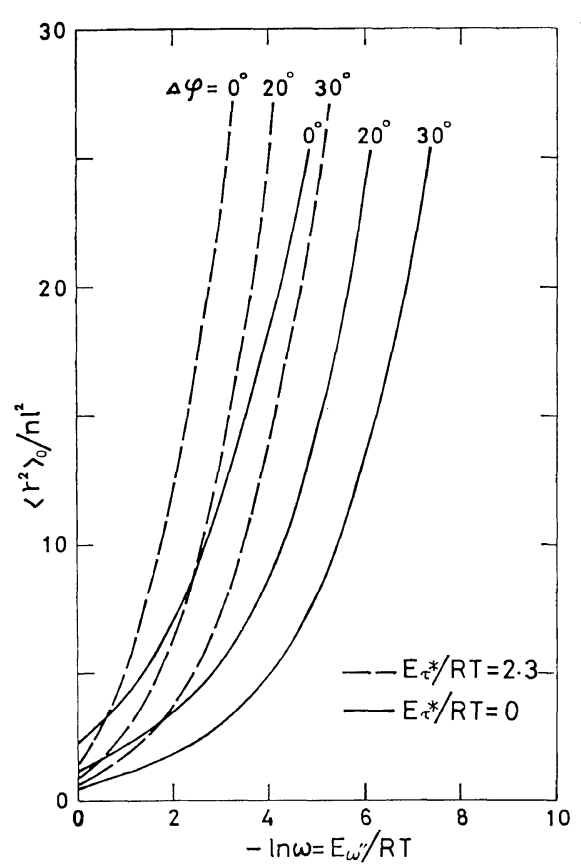

Figure 3. Variation of the characteristic ratio $\left\langle r^{2}\right\rangle_{0} / n l^{2}$ with $-\ln \omega^{\prime \prime}=E_{\omega^{\prime \prime}} / R T$ for the isotactic configuration. Calculated for polymer chains of infinite length with parameters as indicated.

$\tau^{*}$ on the stereoregular syndiotactic chain configuration is illustrated in Figure 4, where again the slope becomes more positive in the reasonable range of $E_{\omega^{\prime \prime}} / R T$ as $\tau^{*}$ decreases. With sufficiently large values of $E_{\omega^{\prime \prime}} / R T$, however, the characteristic ratio reaches an asymptotic value. When $\tau^{*}<1$ for polymers with $\mathrm{R}=\mathrm{CH}_{2} \mathrm{CH}_{3}$ or $\mathrm{CH}_{2} \mathrm{CH}_{2} \mathrm{CH}_{3}$, following the discussion presented in the previous section, here and elsewhere we let $\Delta \varphi_{t}=0$ for a pair of consecutive bonds like $i-1$ and $i$, both being simultaneously in the trans conformation. In other words, we assume here simply that such $(t, t)$ state is most stable when the associated bonds from $i-2$ to $i+1$ are literally in the planar zigzag conformation. For an isotactic sequence, the transition between the two preferred helical conformations is permitted through this arrangement, i.e., . . $\left(t g^{\mp}\right)(t t)\left(g^{ \pm} t\right) \ldots$. In the reasonable range of $E_{\omega^{\prime \prime}} / R T$, the effect is negligibly small in an isotactic chain because the occurrence of such state is quite infrequent. It can be predicted, however, from the conformational analysis that the relative importance of such

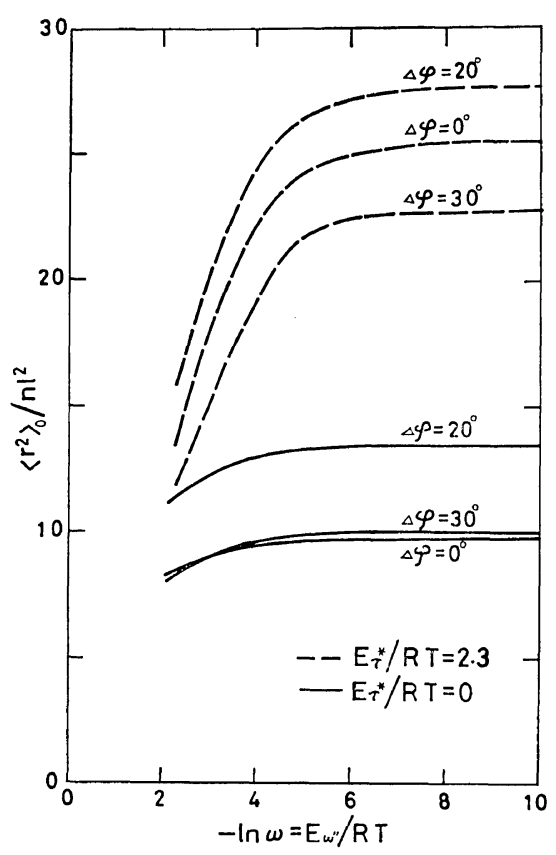

Figure 4. The characteristic ratio vs. $-\ln \omega^{\prime \prime}=$ $E_{\omega^{\prime \prime}} / R T$ for the syndiotactic configuration. Calculated for chain sequences of 100 monomer units $(n=200)$.

conformation increases as the chain configuration becomes more syndiotactic. Consequently the asymptotic value of $\left\langle r^{2}\right\rangle_{0} / n l^{2}$ shown in Figure 4 for $E_{\tau} * / R T=2.3$ should be higher by $c a .10$ in both cases when $\Delta \varphi=20^{\circ}$ and $30^{\circ}$, if we let $\Delta \varphi_{t}=\Delta \varphi$ for bonds $i-1$ and $i$ regardless of the rotational states of the adjoining bonds. Since our interests are mainly focussed in the range of isotactic to atactic polymer configurations, the effect of such revision is comparatively unimportant. Thus we shall not pursue this point further.

Variation of $\left\langle r^{2}\right\rangle_{0} / n l^{2}$ as a function of $-\ln \tau^{*}=$ $E_{\tau} * / R T$ is shown in Figure 5 for $E_{\omega^{\prime \prime}} / R T=5$ and 3 , respectively. Here all the calculations are carried out with $\Delta \varphi=20^{\circ}$. The initial decrease of $\left\langle r^{2}\right\rangle_{0} / n l^{2}$ with $E_{\tau} * / R T$ for chains of the syndiotactic arrangement is mainly due to the arbitrary choice of $\Delta \varphi_{t}$ associated with the aforesaid $(t, t)$ conformation in the neighborhood of $\tau^{*}=1.0$. When $\tau^{*}<0.5$, we let such $\Delta \varphi_{t}=$ 0 . It is implied here that the thermal coefficient of $\left\langle r^{2}\right\rangle_{0}$ for a stereoregular chain, either isotactic or syndiotactic, becomes drastically negative as 


\section{A. ABE}

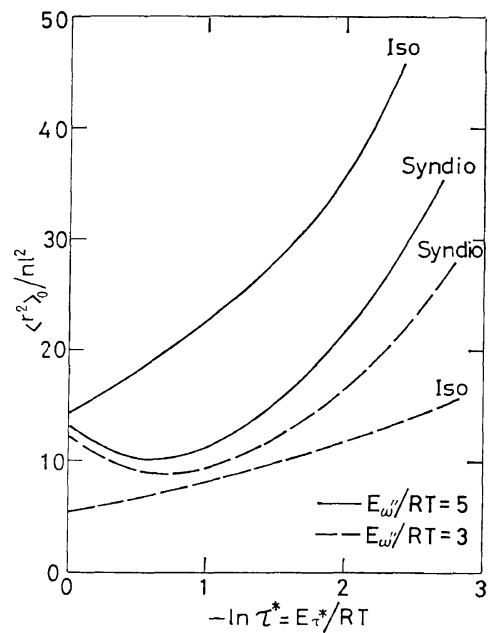

Figure 5. Variation of the characteristic ratio with $-\ln \tau^{*}=E_{\tau} * / R T$ for the stereoregular isotactic $(n=\infty)$ and syndiotactic $(n=200)$ systems. The curves derived for $\Delta \varphi=20^{\circ}$ are shown.

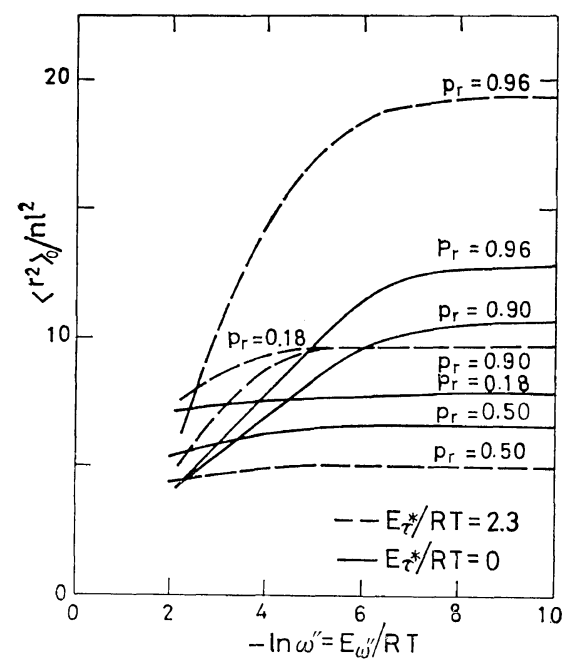

Figure 6. The characteristic ratio vs. $-\ln \omega^{\prime \prime}=$ $E_{\omega^{\prime \prime}} / R T$ for stereoirregular chain systems. Calculated for $\tau^{*}=1$ and 0.1 , respectively, $\Delta \varphi$ being kept at $20^{\circ}$.

$\tau^{*}$ decreases, owing to the negative contribution from $\partial \ln \left\langle r^{2}\right\rangle_{0} / \partial \ln \tau^{*}$ in addition to that ascribable to $\partial \ln \left\langle r^{2}\right\rangle_{0} / \partial \ln \omega^{\prime \prime}$. Such consideration is, however, obviously inconsistent with the experimental observations on the "isotactic" samples of poly-1-butene or poly-1-pentene ${ }^{6}$, for which we may assume $E_{\tau} * / R T \cong 2$.
The effect of $\tau^{*}<1$ on the unperturbed dimension of polymer chains having stereoirregular repeating units is illustrated in Figure 6, where $\left\langle r^{2}\right\rangle_{0} / n l^{2}$ calculated for $\Delta \varphi=20^{\circ}$ is plotted against $-\ln \omega^{\prime \prime}=E_{\omega^{\prime \prime}} / R T$. Here, in keeping with the previous usage ${ }^{1}$, we define $p_{r}$ as the probability that the stereochemical configuration of any given unit is identical with that of the predecessor in the chain. Such chain configuration can be determined by a series of random numbers generated in the range $0-1$. The replication probability $p_{r}$ thus defined may be also regarded as the probability of an isotactic dyad placement. Stereoirregularity prescribed in this manner predominantly comprises block character as indicated by ...ddll. . or vice versa. For a later purpose, we designate chain arrangements which follow such statistics as type I. In Figure 7, the characteristic ratio $\left\langle r^{2}\right\rangle_{0} / n l^{2}$ is plotted against $-\ln \tau^{*}=E_{\tau} * / R T$ for given values of $E_{\omega^{\prime \prime}} / R T$. It was pointed out previosuly ${ }^{1}$ that the condition $\tau^{*}<1$ is important in understanding the small positive temperature coefficient $d \ln \left\langle r^{2}\right\rangle_{0} / d T$ of atactic polymer systems with $\mathrm{R}=\mathrm{CH}_{2} \mathrm{CH}_{3}$ or $\mathrm{CH}_{2} \mathrm{CH}_{2} \mathrm{CH}_{3}$. In the region of atactic configuration, i.e., $p_{r}=c a$. 0.5, the contribution from $\partial \ln \left\langle r^{2}\right\rangle_{0} / \partial \ln \tau^{*}$ becomes slightly positive, while the coefficient $\partial \ln \left\langle r^{2}\right\rangle_{0} /$ $\partial \ln \omega^{\prime \prime}$ diminishes to zero. When $p_{r}$ approaches either the isotactic $\left(p_{r}=1.0\right)$ or the syndiotactic configuration $\left(p_{r}=0.0\right)$, the slope of the curve

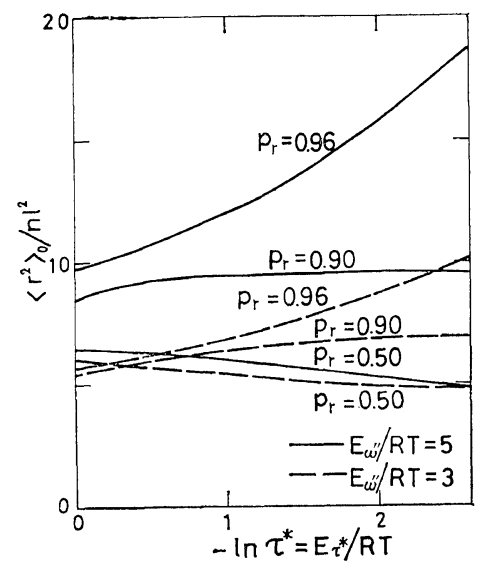

Figure 7. The characteristic ratio vs. $-\ln \tau^{*}=$ $E_{\tau} * / R T$ for stereoirregular chain systems. The value of $\Delta \varphi$ is set equal to $20^{\circ}$ in conformity with the presentation in Figure 6 . 


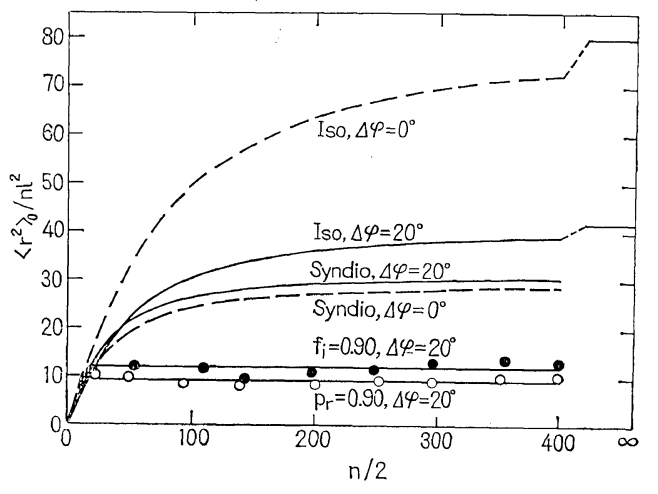

Figure 8. Asymptotic behavior of the characteristic ratio with degree of polymerization. Calculated for isotactic, moderately isotactic and syndiotactic chains using parameters $E_{\tau} * / R T=2.3, E_{\omega^{\prime \prime}} / R T=5$ and $\Delta \varphi$ as indicated. The notations $p_{r}$ and $f_{i}$ are used to represent stereoregularity of the polymer chain systems designated as type I and II, respectively.

shown in Figure 7 becomes more positive, thus leading to highly negative values of $\partial \ln \left\langle r^{2}\right\rangle_{0} /$ $\partial \ln \tau^{*}$.

Asymptotic behaviors of $\left\langle r^{2}\right\rangle_{0} / n l^{2}$ with the polymer chain length is given in Figure 8 , for the isotactic, moderately isotactic, and syndiotactic configurations, respectively. Calculations were carried out for $E_{\tau} * / R T=2.3$ and $E_{\omega^{\prime}} / R T=5$. The effect of $\Delta \varphi$ is to displace the asymptotic values of $\left\langle r^{2}\right\rangle_{0} / n l^{2}$, in both isotactic and syndiotactic chains. Introduction of small portions of less stereoregular placements affect markedly the asymptote. Results are shown in Figure 8 for a chain with $p_{r}=0.9$. The values of $\left\langle r^{2}\right\rangle_{0} /$ $n l^{2}$, as indicated by $\bigcirc$, remain invariable within a certain range of scatter at any degree of polymerization from oligomer to polymer.

Recently Cossee and $\operatorname{Arlman}^{21}$ proposed a polymerization mechanism which may predominantly yield stereoirregular placements of the type ...dld ... or ...ldl... dispersed randomly among $d$ or $l$ sequences, respectively. Polymer chains of this sort will be hereafter called type II to make distinction from the previous one. Chain characteristics of type II have been examined for the optical rotatory behavior in a previous paper $^{8}$. In the highly isotactic region, configurational aspects of such chains are quite different from those of type I. The difference tends to be indistinguishable, however, as the polymer configuration becomes atactic or highly syndiotactic. An example for such configuration is included in Figure 8. The curve derived for a chain having an isotactic dyad fraction $f_{i}=0.9$, each point being indicated by $\bullet$, shows quite a similar behavior to that for the polymer sequence type I.

In Figure 10 of the previous paper ${ }^{1}$, we have plotted the asymptotic value of $\left\langle r^{2}\right\rangle_{0} / n l^{2}$ for $\omega^{\prime \prime}=0$ against $p_{r}$. Similar plots, but for different values of the conformational energy, are presented in Figure 9 for the polymer system with $\mathrm{R}=\mathrm{CH}_{3}$. In Figure 9a, the results obtained for $E_{\omega^{\prime \prime}} / R T=$ $4 \pm 1, \Delta \varphi$ being kept at $20^{\circ}$ are shown, while in Figure $9 \mathrm{~b}$, higher values of conformational energy $\left(E_{\omega^{\prime \prime}} / R T=5 \pm 1\right)$ with larger displacement of the rotational angle $\left(\Delta \varphi=30^{\circ}\right)$ were employed. Similar calculations carried out for the system with $\mathrm{R}=\left(\mathrm{CH}_{2}\right)_{z} \mathrm{CH}_{3} \quad(z \geqq 1)$ are summarized in Figure 10, where the parameters used are $E_{\tau} * / R T=2.3, E_{\omega^{\prime}} / R T=5 \pm 1$ and $\Delta \varphi=20^{\circ}$. Comparison between these two figures indicates that dependence of $\left\langle r^{2}\right\rangle_{0} / n l^{2}$ on the stereoregularity is more sensitive in the latter system. This may be taken as a reflection of the fact that the polymer chain becomes stiffer as $\tau^{*}$ decreases. The temperature coefficients $d \ln \left\langle r^{2}\right\rangle_{0} / d \ln T$ were evaluated according to Eq. 11 , with $E_{\omega^{\prime \prime}} \mid$ $R T=4$ for $\mathrm{R}=\mathrm{CH}_{3}$, and with $E_{\tau} * / R T=2.3$ and $E_{\omega^{\prime \prime}} / R T=5$ for $\mathrm{R}=\left(\mathrm{CH}_{2}\right)_{z} \mathrm{CH}_{3} \quad(z \geqq 1)$, respectively. The temperature coefficients thus derived were found to be quite insensitive to values of $\Delta \varphi$ in the range $0-30^{\circ}$, notwithstanding that the chain dimension largely depends on $\Delta \varphi$. The results are shown in Figures 11 and 12 as a function of the stereoregularity of the chain system.

Some of the results computed for polymer sequences of type II in the moderately isotactic region are also included in Figures 9-12. In polymer systems in which the isotactic sequence is occasionally interrupted by inversion of configuration, the end-to-end dimension as well as its temperature coefficient varies less sharply with isotacticity as expressed by the fraction of isotactic dyads, $f_{i}$. Such tendency becomes more marked as $\tau^{*}$ decreases. The general trend over the entire range of the tacticity is unchanged, however, by such modification of the chain configuration.

Calculations were further extended to the 

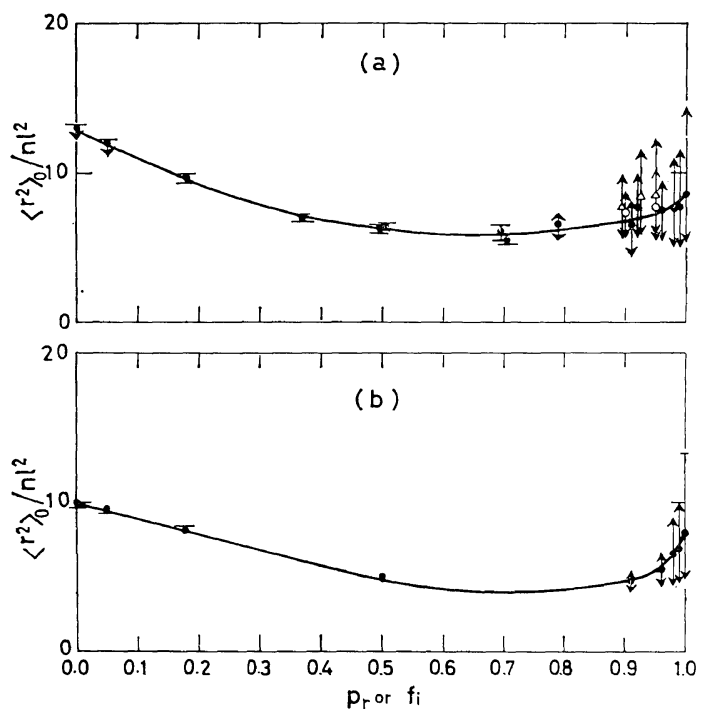

Figure 9. Variation of characteristic ratio with stereoregularity as expressed by $p_{r}$ or $f_{i}$, calculated for the system with $\mathrm{R}=\mathrm{CH}_{3}$. The parameters used are (a) $E_{\omega^{\prime \prime}} / R T=4 \pm 1$ and $\Delta \varphi=20^{\circ}$, and (b) $E_{\omega^{\prime \prime}} / R T=5 \pm 1$ and $\Delta \varphi=30^{\circ}$, respectively. Alteration of $\left\langle r^{2}\right\rangle_{0} / n l^{2}$ within the range of $E_{\omega^{\prime \prime}} / R T$ investigated is indicated by arrows. Solid and open circles represent values of $\left\langle r^{2}\right\rangle_{0} / n l^{2}$ for chains of type I with 100 and 400 monomer units, respectively. Some of the results obtained for chains of type II, comprising 400 monomer units, are also included $(\triangle)$.

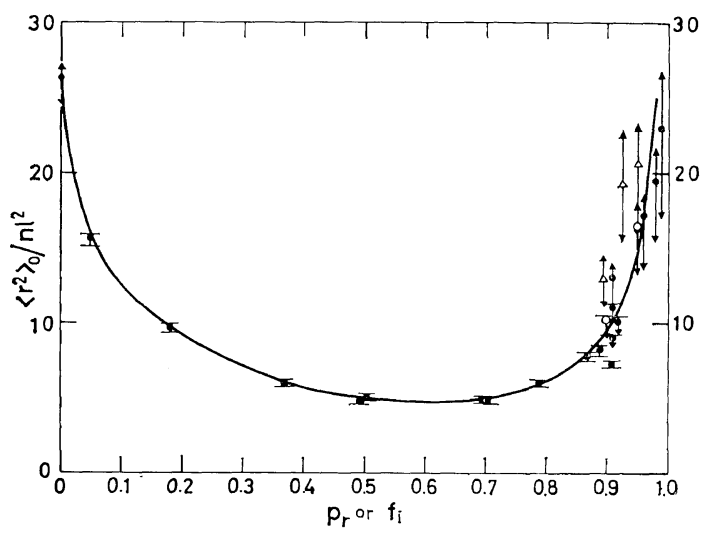

Figure 10. The characteristic ratio as a function of stereoregularity, calculated for the system with $\mathrm{R}=$ $\left(\mathrm{CH}_{2}\right)_{z} \mathrm{CH}_{3}(z \geqq 1)$. The parameters used are $E_{\tau} * 1$ $R T=2.3, E_{\omega^{\prime \prime}} / R T=5 \pm 1$ and $\Delta \varphi=20^{\circ}$, respectively. For description of the marks, see the legend to Figure 9.

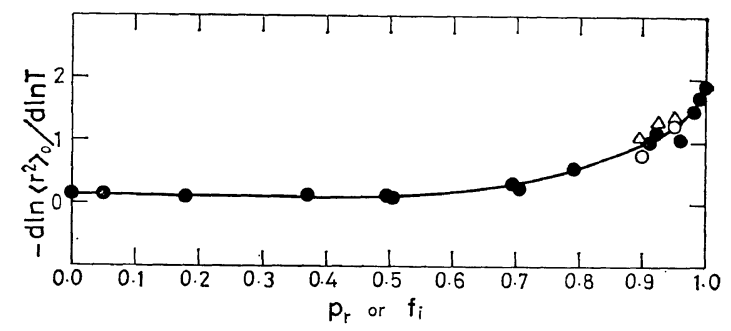

Figure 11. The temperature coefficient $-d \ln \left\langle r^{2}\right\rangle_{0} / d$ In $T$ as a function of stereoregularity, estimated for the polymer system with $\mathrm{R}=\mathrm{CH}_{3}$ by using parameters $E_{\omega^{\prime \prime}} / R T=4$ and $\Delta \varphi=20^{\circ}$, respectively. The results obtained for Monte Carlo chains of type I are shown by solid circles for 100 monomer units and by open circles for 400 monomer units, respectively, and those for chains of type II with 400 monomer units by $\triangle$.

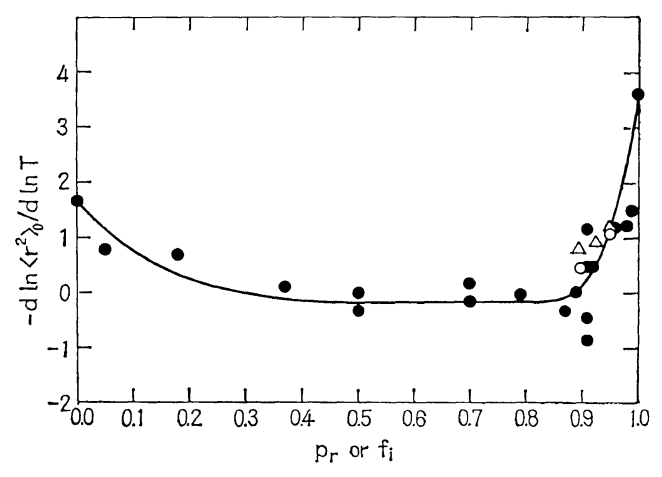

Figure 12. The temperature coefficient of $\left\langle r^{2}\right\rangle_{0}$ as a function of stereoregularity, calculated for the system with $\mathrm{R}=\left(\mathrm{CH}_{2}\right)_{z} \mathrm{CH}_{3}(z \geqq 1)$ by using parameters $E_{\tau} * / R T=2.3, E_{\omega^{\prime \prime}} / R T=5$ and $\Delta \varphi=20^{\circ}$, respectively. For description of the marks, see the legend to Figure 11.

polymer system where the assumption $\tau^{*} \cong \omega^{\prime \prime}$ is adequate. The results obtained for $E_{\tau} * / R T=$ $E_{\omega^{\prime \prime}} / R T=5$ are shown by the dotted curves in Figures 13 and 14 . For such a polymer with stereoregular configuration, either isotactic or syndiotactic, theory predicts a large extension of the chain and a definitely negative temperature coefficient. The dimension of the polymer decreases rapidly as soon as the chain configuration departs from these regular arrangements. In the less stereoregular region, the temperature coefficient $d \ln \left\langle r^{2}\right\rangle_{0} / d \ln T$ may be positive or 


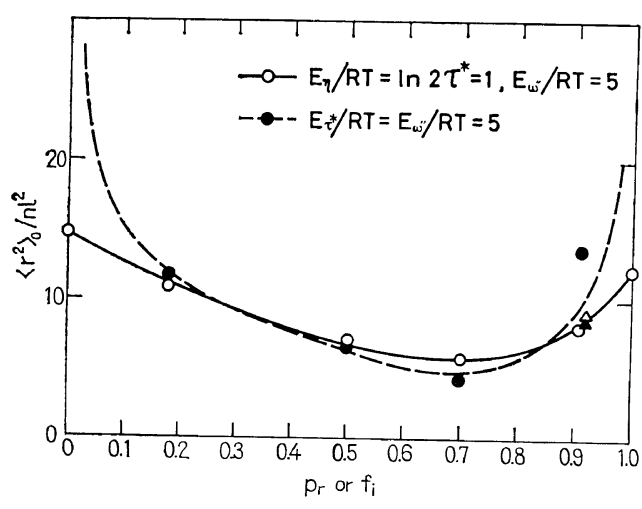

Figure 13. The characteristic ratio vs. stereoregularity for the polymer system (100 monomer units) with $\mathrm{R}=\mathrm{CH}_{2} \mathrm{CH}\left(\mathrm{CH}_{3}\right)_{2}$ or cylohexyl (O) and $\mathrm{R}=$ phenyl ( $\bigcirc)$, respectively. Values of $\left\langle r^{2}\right\rangle_{0} / n l^{2}$ obtained for a chain of type II at $f_{i}=\mathbf{0 . 9 2}$ are separately shown by $\Delta$ and $\triangle$, correspondingly. The value of $\Delta \varphi$ is set equal to $20^{\circ}$, throughout.

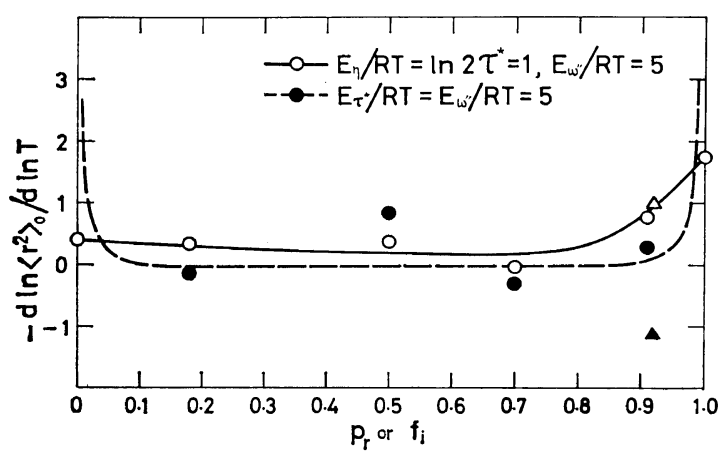

Figure 14. The temperature coefficient of $\left\langle r^{2}\right\rangle_{0}$ for the polymer systems treated in Figure 13. For description of the marks, see the legend to Figure 13. Since we adopted the expression $\tau^{*}=0.5 \exp \left(E_{\eta} / R T\right)$ for polystyrene as given by Williams and Flory ${ }^{19}$, the second term on the right hand side of Eq. 11 for the temperature coefficient should then be necessarily replaced by $-\ln 2 \tau^{*}\left(\partial \ln \left\langle r^{2}\right\rangle_{0} / \partial \ln \tau^{*}\right)$.

negative depending upon the chain configuration. For comparison, calculations with the statistical weight parameters deduced for polystyrene ${ }^{19}$ are also shown in Figures 13 and 14 . Geometrical properties of this polymer have been treated with the assumption $\omega^{\prime \prime}=0$ by Flory ${ }^{22}$. It should be noted here that the role of $\tau^{*}$ becomes conspicuously important only in the stereoregular chain system.

\section{COMPARISON WITH EXPERIMENTAL OBSERVATIONS}

According to a recent report by Nakajima and Saijo $^{10}$, viscometric determination in a series of theta solvents yielded the characteristic ratio* $\left\langle r^{2}\right\rangle_{0} / n l^{2}=6.4$ to 4.7 , and the thermal coefficient ${ }^{* *}$ $d$ ln $\left\langle r^{2}\right\rangle_{0} / d T \cong-4 \times 10^{-3} \mathrm{deg}^{-1}$ over the temperature range from 125 to $183^{\circ} \mathrm{C}$. These data agree well with that reported some time ago by Kinsinger and Hughes ${ }^{23}$. Some chain characteristics consistent with $\left\langle r^{2}\right\rangle_{0} / n l^{2} \cong 6$ are tabulated in Table I for "highly" isotactic chains. The conventional isotactic polypropylene samples available for experiments may find their equivalent within the range $\Delta \varphi=20-30^{\circ}, E_{\omega^{\prime \prime}} / R T=3$ -4 , and $p_{r}$ (or $f_{i}$ ) $=0.92-0.98$. The large negative temperature coefficients given in Table I are in the same order of magnitude as that reported by Nakajima et $a .^{10}$ By judging from the nature of polymerization, assumption of the perfect isotacticity (i.e., $p_{r}=1.0$ ) is rather unrealistic. As a matter of fact, according to a recent communication from Hamada and Flory ${ }^{24}$, determination of the intrinsic viscosity of the isotactic polypropylene in decalin as a function of temperature led to $d \ln \left\langle r^{2}\right\rangle_{0} / d T=-1.6$

Table I. Estimation of chain characteristics of isotactic polypropylene consistent with $\left\langle r^{2}\right\rangle_{0} / n l^{2}=6.0$

\begin{tabular}{lcccc}
\hline $\begin{array}{c}p_{r} \\
\text { (or } f_{i} \text { ) }\end{array}$ & $\begin{array}{c}\text { Type of } \\
\text { the chain }\end{array}$ & $\Delta \varphi\left(^{\circ}\right)$ & $E_{\omega^{\prime \prime}} / R T$ & $\begin{array}{c}-d \ln \left\langle r^{2}\right\rangle_{0} \\
d T \times 10^{3} \\
\left(\mathrm{deg}^{-1}\right)\end{array}$ \\
\hline 1.0 & & 20 & 3.3 & 3.9 \\
& & 30 & 4.4 & 5.3 \\
0.98 & I & 20 & 3.4 & 3.7 \\
& & 30 & 4.7 & 4.0 \\
0.96 & I & 20 & 3.2 & 2.7 \\
& & 30 & 5.1 & 2.4 \\
0.95 & II & 20 & 3.1 & 3.0 \\
0.925 & II & 20 & 3.1 & 2.8 \\
0.91 & I & 20 & 3.7 & 2.8 \\
0.895 & II & 20 & 3.3 & 2.8 \\
\hline
\end{tabular}

* For consistency, we adopt values of $\left\langle r^{2}\right\rangle_{0} / n l^{2}$ which are derived using the constant $\Phi=2.5 \times 10^{21}$ associated with the viscosity-molecular weight relation.

** Although the thermal coefficient derived in this manner may be affected by the probable solvent effect, a large negative value is inevitably implied. 
$( \pm 0.2) \times 10^{-3} \mathrm{deg}^{-1}$. In this measurement, special care was taken to avoid the possible oxidative degradation of the sample at higher temperatures, which may of course enhance erroneously the magnitude of the temperature coefficient.

Experimental data ${ }^{23,25}$ on atactic polypropylene observed in various theta solvents at 34, 74 and $92^{\circ} \mathrm{C}$ seem to indicate $\left\langle r^{2}\right\rangle_{0} / n l^{2} \cong 7$ with a fairly small temperature coefficient. Inagaki, Miyamoto and $\mathrm{Ohta}^{26}$ reported the characteristic ratio for the syndiotactic analog to be 6.9 at $45^{\circ} \mathrm{C}$. As shown in Figure 9, theoretical calculations with parameters chosen from the aforementioned range would give $\left\langle r^{2}\right\rangle_{0} / n l^{2}=6$ to 7 for atactic chains $\left(p_{r}=0.8-0.3\right)$. However, the value $\left\langle r^{2}\right\rangle_{0} / n l^{2}$ tends to increase further toward the syndiotactic configuration as $p_{r}$ decreases beyond this region. For a perfectly syndiotactic chain, we have $\left\langle r^{2}\right\rangle_{0} / n l^{2}=10$ to 13 . A quantitative comparison between the theoretical calculation and the experiment is improbable since the tacticity of the "syndiotactic" polymer is not known accurately ${ }^{26}$.

Experimental values ${ }^{5,6}$ of $\left\langle r^{2}\right\rangle_{0} / n l^{2} \cong 10$ and $d \ln \left\langle r^{2}\right\rangle_{0} / d T \cong 0$ for isotactic polybutene-1 and polypentene-1 may be found to fit our model calculations at $p_{r}=0.90-0.93$ with $\Delta \varphi=20^{\circ}$, $E_{\tau} * / R T \cong 2.3$ and $E_{\omega^{\prime \prime}} / R T \cong 5$ for the polymer sequence type I (cf. Figures 10 and 12). For the chain sequence type II, the agreement with the observed values would be obtained at $f_{i} \cong 0.85$ when the other parameters are kept invariable. With $E_{\omega^{\prime}} / R T=3$, the temperature coefficient should be still largely negative at $p_{r}=0.90$ or at $f_{i}=0.85$ where $\left\langle r^{2}\right\rangle_{0} / n l^{2} \cong 10$. The agreement should not be improved by varying $\Delta \varphi$ since the temperature coefficient is quite insensitive to such modifications in the range $0-30^{\circ}$.

Viscometric studies on atactic polybutene- 1 in various theta solvents were reported by Moraglio, Gianotti and Danusso ${ }^{27}$, who found the value $\left\langle r^{2}\right\rangle_{0} / n l^{2}$ to be $c a .7$ at ordinary temperatures. The temperature coefficient estimated therein, $d \ln$ $\left\langle r^{2}\right\rangle_{0} / d T=-1.2 \times 10^{-3} \mathrm{deg}^{-1}$, was appreciably negative and at variance with that $\left(0.5 \times 10^{-3}\right.$ $\mathrm{deg}^{-1}$ ) reported from thermoelastic measurements at somewhat higher temperatures $\left(140-200^{\circ} \mathrm{C}\right)$ by Mark and Flory ${ }^{6}$. On the other hand, theoretical calculations suggest $\left\langle r^{2}\right\rangle_{0} / n l^{2}=6$ to 7 for $p_{r}=0.80-0.85$, and 5 to 6 for $p_{r}=0.4-0.8$ with zero or slightly positive temperature coefficients ( $c f$. Figures 10 and 12). Although we do not know exactly what is meant by the term "atactic", there remains some contradiction between the theoretical calculations and the experimental results derived from the dilute solution properties. As we have postulated in our theoretical treatment, the difference between polymers of butene- 1 and pentene- 1 is found to be quite small in the atactic region. A slightly positive temperature coefficient, $d \ln \left\langle r^{2}\right\rangle_{0} / d T=0.53 \times$ $10^{-3} \mathrm{deg}^{-1}$, was reported ${ }^{6}$ for atactic poly-1-pentene from thermoelastic measurements conducted over the temperature range $40-140^{\circ} \mathrm{C}$, whereas Moraglio and Gianotti ${ }^{28}$ estimated the ratio $\left\langle r^{2}\right\rangle_{0} / n l^{2}$ to be ca. 7.2 at $30^{\circ} \mathrm{C}$.

Solution properties of atactic poly(vinylcyclohexane) prepared by hydrogenation of polystyrene were studied by Elias and Etter ${ }^{29}$. The radically prepared polystyrene was suggested to be atactic to moderately syndiotactic ${ }^{30}$. The characteristic ratio $\left\langle r^{2}\right\rangle_{0} / n l^{2}$ of the hydrogenated polystyrene determined under the theta condition was 10.8 at $25^{\circ} \mathrm{C}$, whereas the widely accepted value for atactic polystyrene $\mathrm{e}^{11}$ is $\mathrm{ca} .10$ around $35^{\circ} \mathrm{C}$. The agreement is reasonable between these experimental results and the theoretical calculations presented in Figure 13. It has been suggested ${ }^{31}$ from the measurements in thermodynamically good solvents that the difference in the unperturbed dimensions of isotactic and atactic polystyrene is small. This is also in agreement with the results shown in Figure 13. Some discrepancy may be noted however in the temperature coefficient $d \ln \left\langle r^{2}\right\rangle_{0} / d T$ of polystyrene. While the most reliable experimental value ${ }^{32}$ is $0.4 \times 10^{-3} \mathrm{deg}^{-1}$, a small, but definitely negative temperature coefficient is suggested from the present treatment.

\section{DISCUSSION}

Natta and Corradini ${ }^{7}$ have emphasized the preference of the polymer sequence designated with type II over that of type I from the crystallographic consideration. The mean persistence length of isotactic arrangements is longer in the former system when compared at a given value of isotactic dyad content. To find agreement 
with the observed temperature coefficient $d \ln$ $\left\langle r^{2}\right\rangle_{0} / d T \cong 0$ for highly isotactic poly-1-butene and poly-1-pentene, however, one must get down to a somewhat lower value of isotacticity for the chain sequence type II. The existing experimental data are not sufficiently decisive on the preference between these stereochemical arrangements.

Adoption of the value $\left\langle r^{2}\right\rangle_{0} / n l^{2} \cong 6$ for highly isotactic polypropylene inevitably implies a large negative thermal coefficient of $\left\langle r^{2}\right\rangle_{0}$ in this system. With the information available at present for the solution properties, however, we cannot make clear decisions on the tacticity of the "isotactic" polypropylene sample beyond those suggested in Table I. In the case of poly-1-butene or poly1-pentene, on the other hand, theoretical calculations and experimental observations give agreement for a chain with $p_{r}=0.90-0.93$ or $f_{i} \cong 0.85$. There seems to be no reason why we should assume exceptionally high isotacticity (i.e., $p_{r} \cong 1.0$ ) only in the case of polypropylene.

The conformational energy $E_{\omega^{\prime \prime}}=3$ to 4 $\mathrm{kcal} \cdot \mathrm{mol}^{-1}$ for polymers with $\mathrm{R}=\left(\mathrm{CH}_{2}\right)_{2} \mathrm{CH}_{3}$ $(z \geqq 1)$ is somewhat higher than that ( 2 to $3 \mathrm{kcal}$. $\mathrm{mol}^{-1}$ ) suggested previously ${ }^{8}$ from the study of the optical rotatory properties of hydrocarbon polymers. Geometrical properties such as the end-to-end dimension should reflect more sensitively the cooperativity in the vinyl polymer system, characterized by interdependence of neighboring bond rotations. Taking $E_{\omega^{\prime \prime}}<3 \mathrm{kcal} \cdot \mathrm{mol}^{-1}$, the coefficient $d \ln \left\langle r^{2}\right\rangle_{0} / d T$ becomes definitely negative even at $p_{r} \cong 0.9$. In this respect, choice of $\tau^{*}$ is not very critical within the range $\tau^{*}=0.1$

-0.2 or alternatively $E_{\tau} * / R T=2.3-1.6$.

The effect of $\tau^{*}$ on the unperturbed dimension is explicitly shown in the perfectly stereoregular, either isotactic or syndiotactic system. Properties estimated for a series of polymers are summarized in Table II, where energetic parameters are chosen so as to represent roughly the system given in the first column. As the side chain becomes bulkier from $\mathrm{R}=\mathrm{CH}_{3}$ to $\mathrm{CH}_{2} \mathrm{CH}\left(\mathrm{CH}_{3}\right)_{2},\left\langle r^{2}\right\rangle_{0} / n l^{2}$ as well as the magnitude of $d \ln \left\langle r^{2}\right\rangle_{0} / d \ln T$ increases drastically. The role of four-bond interactions represented by $\omega^{\prime \prime}$ has been throughly explored at a fixed value of $\tau^{*}$ by Fujiwara and Flory ${ }^{33}$. Preparation and characterization of the perfectly isotactic vinyl polymers with bulky side chains are full of interest.

Stiffness of a polymer chain, as defined by the number of conformations permitted to the system, is primarily determined by the bulkiness of the side groups and the stereochemical configuration along the chain. For an illustration, the conformational partition function $Z$ was evaluated for a chain comprising 100 monomeric residues according to the prescription given by Eq. 11 of ref. 1 . The importance of the $(t, t)$ element of $U^{\prime}$, as it represents the side chain effect, is manifestly shown in Figure 15, where $\log { }_{10} Z$ is plotted against $p_{r}$. In the polymer system where bond rotations are utmost hindered, geometrical properties are, for the most part, determined by the stereochemical configuration of the chain.

Table II. Unperturbed dimension and its temperature coefficient for stereoregular vinyl polymer systems ${ }^{a}$

\begin{tabular}{|c|c|c|c|c|c|c|}
\hline \multirow{2}{*}{$\mathrm{R}$} & \multirow{2}{*}{$E_{\tau} * / R T$} & \multirow{2}{*}{$E_{\omega^{\prime \prime}} / R T$} & \multicolumn{2}{|c|}{ Isotactic } & \multicolumn{2}{|c|}{ Syndiotactic } \\
\hline & & & $\left\langle r^{2}\right\rangle_{0} / n l^{2}$ & $-d \ln \left\langle r^{2}\right\rangle_{0} / d \ln T$ & $\left\langle r^{2}\right\rangle_{0} / n l^{2}$ & $-d \ln \left\langle r^{2}\right\rangle_{0} / d \ln T$ \\
\hline $\mathrm{CH}_{3}$ & 0 & 4 & 8.5 & 1.9 & 13 & 0.1 \\
\hline $\begin{array}{c}\mathrm{CH}_{2} \mathrm{CH}_{3} \\
\quad \text { and }\end{array}$ & 1.4 & 5 & 27 & 3.0 & 14 & 0.9 \\
\hline analogs & 2.3 & 5 & 42 & 3.6 & 26 & 1.7 \\
\hline $\mathrm{CH}_{2} \mathrm{CH}\left(\mathrm{CH}_{3}\right)_{2}$ & 5 & 5 & $93^{b}$ & $2.2^{\mathrm{b}}$ & $183^{b}$ & $1.7^{\mathrm{b}}$ \\
\hline $\begin{array}{c}\mathrm{C}_{6} \mathrm{H}_{5} \\
\text { (phenyl) }\end{array}$ & $-1^{c}$ & 5 & 12 & 1.5 & 15 & 0.4 \\
\hline
\end{tabular}

a Unless otherwise noted, the asymptotic values for $n=\infty$ or the equivalent are listed. The value of $\Delta \varphi$ is set equal to $20^{\circ}$, for illustrative purposes.

b Calculated for polymer chains comprising 400 monomer units. The asymptotic values $\left(\left\langle r^{2}\right\rangle_{0} / n l^{2}\right)_{n=\infty}$ should be much larger and the corresponding temperature coefficients more negative.

c The statistical weight parameter $\tau^{*}$ is given by $\ln 2 \tau^{*}=-E_{\tau} * / R T$. 


\section{A. $\mathrm{ABE}$}

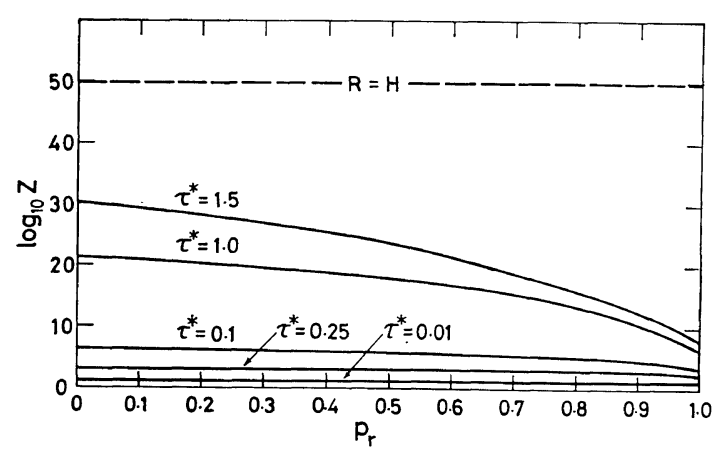

Figure 15. Variation of chain stiffness of the vinyl polymer with stereoregularity. The conformational partition function $Z$ for a chain comprising 100 monomer units was evaluated for a series of $\tau^{*}$ 's, the other parameter $\omega^{\prime \prime}$ being kept constant at 0.01 . A value derived for polyethylene $(\mathrm{R}=\mathrm{H})$ is also shown for comparison by the dotted line; the parameters used are $\sigma=0.5$ and $\omega^{\prime \prime}=0.01$, respectively.

It should be pointed out, finally, that the magnitude of $\left\langle r^{2}\right\rangle_{0} / n l^{2}$ cannot provide a good criterion to define the stiffness of the vinyl chain system except when the polymer is in a specified configuration.

Acknowledgment. The author wishes to thank Prof. P. J. Flory of Stanford University for his unflagging encouragement.

\section{REFERENCES}

1. P. J. Flory, J. E. Mark, and A. Abe, J. Amer. Chem. Soc., 88, 639 (1966).

2. A. Zambelli, A. L. Segre, M. Farina, and G. Natta, Makromol. Chem., 110, 1 (1967).

3. P. J. Flory and J. D. Baldeschwieler, J. Amer. Chem. Soc., 88, 2873 (1966).

4. P. J. Flory and Y. Fujiwara, Macromolecules, 2, 315, 327 (1969).

5. (a) G. Moraglio and J. Brzezinski, J. Polym. Sci., Part B, 2, 1105 (1964); (b) K. S. Sastry and R. D. Patel, Europ. Polymer J., 5, 79 (1969).

6. J..E. Mark and P. J. Flory, J. Amer. Chem. Soc., 87, 1423 (1965).

7. G. Natta and P. Corradini, Makromol. Chem., 110, 291 (1967).

8. A. Abe, J. Amer. Chem. Soc., 90, 2205 (1968).

9. G. Allegra, Makromol. Chem., 117, 24 (1968).

10. A. Nakajima and A. Saijo, J. Polym. Sci., Part A-2, 6, 735 (1968).
11. P. J. Flory, Statistical Mechanics of Chain Molecules (Interscience Publishers, New York, 1969).

12. E. B. Wilson, Jr., Advan. Chem. Phys., 2, 367 (1959).

13. J. B. Hendrickson, J. Amer. Chem. Soc., 83, 4537 (1961).

14. A. Abe, R. L. Jernigan, and P. J. Flory, ibid., 88, 631 (1966).

15. D. W. Scott, J. P. McCullough, K. D. Williamson, and G. Waddington, ibid., 73, 1707 (1951).

16. J. K. Brown and N. Sheppard, J. Chem. Phys., 19, 976 (1951).

17. E. L. Eliel, N. L. Allinger, S. L. Angyal, and G.A. Morrison, Conformational Analysis (Interscience Publishers, New York, 1965) Chap. 2, p. 43.

18. A. Abe and T. Hama, J. Polym. Sci., Part B, 7, 427 (1969).

19. A. D. Williams and P. J. Flory, J. Amer. Chem. Soc., 91, 3111 (1969).

20. R. L. Miller, "Crystalline and Spherulitic Properties" in Crystalline Olefin Polymers, Part I, R. A. V. Raff and K. W. Doak, Ed. (Interscience Publishers, New York, 1965), p. 577.

21. P. Cossee, J. Catalysis, 3, 80 (1964); E. J. Arlman, ibid., 3, 89 (1964); E. J. Arlman and P. Cossee, ibid., 3, 99 (1964); E. J. Arlman, ibid., 5, 178 (1966).

22. P. J. Flory, Private communication.

23. J. B. Kinsinger and R. E. Hughes, J. Phys. Chem., 67, 1922 (1963).

24. P. J. Flory and F. Hamada, unpublished results.

25. F. Danusso, G. Moraglio, V. Giannella, and G. Natta, Rend. Accad. Naz. Lincei, 25, 510 (1958).

26. H. Inagaki, T. Miyamoto, and S. Ohta, J. Phys. Chem., 70, 3420 (1966).

27. G. Moraglio, G. Gianotti, and F. Danusso, Europ. Polymer J., 3, 251 (1967).

28. G. Moraglio and G. Gianotti, Chim. Ind. (Milan), 49, 927 (1967).

29. H.-G. Elias and O. Etter, J. Macromol. Chem., 1, 431 (1966).

30. S. Brownstein, S. Bywater, and D. J. Worsfold, J. Phys. Chem., 66, 2067 (1962); A. L. Segre, P. Ferruti, E. Toja, and F. Danusso, Macromolecules, 2, 35 (1969); Y. Fujiwara and P. J. Flory, private communication.

31. W. R. Krigbaum, D. K. Carpenter, and S. Newman, J. Phys. Chem., 62, 1586 (1958).

32. T. A. Orofino and A. Ciferri, ibid., 68, 3136 (1964).

33. Y. Fujiwara and P. J. Flory, private communication. 